\title{
Mechanical Properties of Group Iv Single-walled Nanotubes: A Finite Element Approach Based on the Density Functional Theory
}

Mohammad Dastmard

University of Guilan

Reza Ansari ( $\nabla$ r_ansari@guilan.ac.ir)

University of Guilan https://orcid.org/0000-0002-6810-6624

Saeed Rouhi

Azad University: Islamic Azad University

\section{Research Article}

Keywords: Density functional theory, Finite element method, Mechanical properties, Single-walled nanotube, Group IV elements

Posted Date: March 3rd, 2021

DOI: https://doi.org/10.21203/rs.3.rs-250867/v1

License: (a) (1) This work is licensed under a Creative Commons Attribution 4.0 International License. Read Full License 


\title{
Mechanical properties of group IV single-walled nanotubes: a finite element approach based on the density functional theory
}

\author{
M. Dastmard ${ }^{1}$, R. Ansari ${ }^{\star 2}$, S. Rouhi ${ }^{3}$ \\ ${ }^{1}$ Faculty of Mechanical Engineering, University Campus 2, University of Guilan, Rasht, Iran \\ ${ }^{2}$ Faculty of Mechanical Engineering, University of Guilan, P.O. Box 3756, Rasht, Iran \\ ${ }^{3}$ Department of Mechanical Engineering, Langarud Branch, Islamic Azad University, \\ Langarud, Iran
}

\begin{abstract}
In this article, the density functional theory is applied to investigate the mechanical properties of single-walled nanotubes of group IV of periodic table including carbon nanotube, silicon nanotube, germanium nanotube and stanene nanotube. $(10,10)$ armchair nanotube is selected for the investigation. By establishing a link between potential energy expressions in the molecular and structural mechanics, a finite element approach is proposed for modeling the nanotubes. In the proposed model, the nanotubes are considered as an assemblage of beam elements. Young's modulus of the nanotubes is computed by the proposed finite element model. Young's modulus of carbon, silicon, germanium, and tin nanotubes are obtained as $1029,159.82,83.23$ and $83.15 \mathrm{GPa}$ respectively, using the density functional theory. Also, the finite element approach gives the values as 1090, 154.67, 85.2 and $82.6 \mathrm{GPa}$ respectively. It is shown that the finite element model can predict the results of the density functional theory with a good accuracy.
\end{abstract}

Keywords: Density functional theory; Finite element method; Mechanical properties; Singlewalled nanotube; Group IV elements

\section{Introduction}

The discovery of carbon nanotubes led to a breakthrough in science and technology [1]. Single-walled carbon nanotubes (SWCNT) were synthesized by Bethune et al. [2] and Iijima and Ichihashi [3] using the arc-discharge methods. Ever since then, the nanotubes structures have been attracting researchers' attention in nanoscience communities.

\footnotetext{
${ }^{*}$ Corresponding authors:

E-mail addresses: r_ansari.guilan.ac.ir
} 
Great properties, including high strength and stiffness, in the range of $\mathrm{TPa}$, ability to withstand large elastic strain, and low density are reported for CNTs [4]. Obtaining mechanical properties, including elastic modulus of CNT, has been one of the most challenging topics in these researches. The structural parameters of CNTs influence their elastic parameters. After a comprehensive review on Young's modulus of CNTs reported in the literature, different values were observed. However, all of them report the same magnitude order for Young's modulus. Treacy et al. [5] reported Young's modulus of CNTs as the average amount of $1.8 \mathrm{TPa}$ using the relation between the Young's modulus and the vibrational energy. The mean value of the Young's modulus was reported as $1.25 \mathrm{TPa}$ by Krishnan et al. [6]. They estimated the stiffness of SWCNTs by observing freestanding vibration modes which are driven stochastically in a transmission electron microscope. Besides, Salvetat et al. [7] calculated an average of 0.81 TPa for CNT Young's modulus.

On the other hand, the theoretical approaches have been employed to calculate Young's modulus of CNTs. Hernandez [8] and Van lier [9] reported the Young's modulus of SWCNTs in range of 1.06 TPA to $1.14 \mathrm{TPa}$ by using ab initio method. Using tight-binding methods, Young's modulus of CNTs was reported in range of $0.676 \mathrm{TPa}$ to $1.27 \mathrm{TPa}[10,11]$. Tserpes et al. [12] used finite element (FE) method to obtain the Young's modulus between $0.95 \mathrm{TPa}$ and 1.05 TPa for armchair, zigzag, and chiral SWCNTs. Kalamkarov et al. [13] calculated Young's modulus of CNTs with the diameters of $4 \AA$ to $35 \AA$ as $0.96 \mathrm{TPa}$ to 1.04 TPa.

Ebrahimi et al. [14] employed density functional theory (DFT) to calculate the response of armchair SWCNT to axial tension. Zang et al. [15] used three different method including DFT, molecular dynamics (MD), and Car-Parrinello molecular dynamics (CPMD) to obtain Young's modulus of SWCNT with the chirality of $(3,3)$. Rafiee et al. [16] considered the influences of diameter and chirality on Young's modulus of CNTs. It was found that Young's modulus of CNT is not dependent on chirality and approximately independent from the CNT diameter. M. Zaeri [17] used MD methodology to obtain the value of $0.943 \mathrm{TPa}$ for Young's modulus of CNTs. Tuan Hung et al. [18] used DFT method to obtain mechanical strength of SWNTs with the diameters in the range of $0.3-0.8 \mathrm{~nm}$. Their calculations showed that for the large SWNTs, Young's modulus does not depend on the CNT diameter and chirality.

After discovery and synthesis of graphene [19], other elements of group-IV in periodic table (including Silicon, Germanium and Stanene) with a graphene-like hexagonal structure were investigated looking for exceptional properties. 
In this regard, silicene, germanene, and stanine, which are tow-dimensional layers made by $\mathrm{Si}, \mathrm{Ge}$, and $\mathrm{Sn}$ atoms, have been predicted [20-26]. Unlike graphene, 2D structure made by silicon, germanium and stanene have a buckled structure [20,22-24,27]. Mortazavi et al. [28] employed first-principle calculations to investigate the mechanical properties of silicene, germanene and stanene nanosheets. The predicted Young's modulus for armchair configuration of silicene, germanene and stanene nanosheets were 61.7, 44 and $25.2 \mathrm{GPa}$ respectively. Besides, Young's modulus of zigzag silicene, germanene and stanene nanosheets are 59, 43.4 and $25.2 G P a$, respectively.

Silicon, Germanium and Tin nanotubes are tube-like nanostructures which are made from Si, Ge and Sn atoms. Byun et al. [29] investigated the responses of hypothetical silicon nanotubes (SiNT) under axial tensile force using an atomistic simulation. They compared the physical values related to the stiffness of SiNTs with those of CNTs and indicated that SiNTs are very brittle. Verma et al. [30] calculated cohesive energy, Young's and shear moduli and Poisson's ratio for SiNT with various diameters and chiralities. They obtained Young's modulus of SiNT is in range of 100-200Gpa. Setoodeh et al. [31] investigated the mechanical properties of Silicon-Germanium nanotubes by MD method. Young's modulus of SiliconGermanium nanotubes with different diameter with constant length of $15 \mathrm{~nm}$ was obtained in the range of 48 to $52 \mathrm{GPa} . \mathrm{nm}$. Abbasi et al. [32] investigated the adsorption of $\mathrm{SO}_{2}$ molecules on the armchair tin nanotube by using DFT calculations.

In this paper, DFT method is utilized to calculate the elastic properties of group IV pristine nanotubes. The results are employed to develop a space frame finite element (FE) model. This mode can be used to evaluate the mechanical properties of the considered. Young's moduli of the nanotubes computed by the proposed model are compared with the results of DFT approach.

\section{Modeling and Computational details}

\subsection{DFT modeling}

DFT calculations are used as implemented in the SIESTA code. Structures are optimized with generalized GGA [33,34]. Besides, PBE exchange correlation is selected [32,33]. Integration of Brillouin zone is carried out with 1x1x40 for the Brillouin zone. Cut-off energy of plane-wave expansion is equal to $450 \mathrm{Ry}$. To optimize the geometry, numerical atomic orbitals are used under DZP (double zeta polarization) basis sets. While, the confinement energy is equal to $50 \mathrm{meV}$. Standard CG (conjugate-gradients) technique is used to perform 
energy minimization. The relaxation is continued until the applied force on all of the atoms be less than $0.04 \mathrm{~d} 0 \mathrm{eV} / \AA$.

Simulated unit cells are represented in Fig. 1. Here, a $(10,10)$ armchair nanotube with 40 atoms is considered for the simulations. Using the explained procedure, the geometrical bond length $(a)$ and buckling height $(\delta)$, see Fig. 2, of the group IV nanotubes are given in Table 1.

\section{FE Modeling}

Odegard et al. [36] developed a FE model for investigation the mechanical characteristics of the CNTs which is composed of beam elements. The elastic modulus of beam elements are obtained by linking the potential energies in the molecular mechanics and structural mechanics. This model was more developed by Li and Chou [37].

The total potential energy of a system in molecular mechanics is determined as the summation of bond stretching energy, $U_{r}$, bond angle bending, $U_{\theta}$, dihedral angle torsion, $U_{\emptyset}$, out-of plane torsion, $U_{\omega}$, and nonbonding van der Waals energies, $U_{v d w}$ [38-39]:

$U_{\text {total }}=\sum U_{r}+\sum U_{\theta}+\sum U_{\emptyset}+\sum U_{\omega}+\sum U_{v d w}$

Here, the $3^{\text {rd }}$ and $4^{\text {th }}$ terms are combined into a single equivalent term. Furthermore, harmonic forms are considered to state remaining terms [40,41]:

$$
\begin{array}{ll}
U_{r}=\frac{1}{2} k_{r}\left(r-r_{0}\right)^{2}=\frac{1}{2} k_{r}(\Delta r)^{2}, & k_{r}=\frac{d^{2} U_{r}}{d \Delta r^{2}} \\
U_{\theta}=\frac{1}{2} k_{\theta}\left(\theta-\theta_{0}\right)^{2}=\frac{1}{2} k_{\theta}(\Delta \theta)^{2}, & k_{\theta}=\frac{d^{2} U_{\theta}}{d \theta^{2}} \\
U_{\tau}=U_{\emptyset}+U_{\omega}=\frac{1}{2} k_{\tau}\left(\tau-\tau_{0}\right)^{2}=\frac{1}{2} k_{\tau}(\Delta \tau)^{2}, & k_{\tau}=\frac{d^{2} U_{\tau}}{d \tau^{2}}
\end{array}
$$

where $k_{r}, k_{\theta}$ and $k_{\tau}$ are the force constants. They are respectively associated with the bond stretching, bond bending angle, and bond torsion angle. The corresponding changes in the bond length, bond bending angle and bond dihedral angle are represented by $\Delta r, \Delta \theta$ and $\Delta \tau$, respectively. If the bonds are considered as beams, their strain energies under tension force $U_{A}$, bending moment $U_{M}$ and torsional loading $U_{\tau}$ are expressed as $[42,43]$ :

$U_{A}=\frac{1}{2} \int_{0}^{L} \frac{F^{2}}{E A} d L=\frac{1}{2} \frac{F^{2} L}{E A}=\frac{1}{2} \frac{E A}{L}(\Delta L)^{2}$ 


$$
\begin{aligned}
& U_{M}=\frac{1}{2} \int_{0}^{L} \frac{M^{2}}{E I} d L=\frac{2 E I}{L}(2 \alpha)^{2}=\frac{1}{2} \frac{E I}{L}(2 \alpha)^{2} \\
& U_{\tau}=\frac{1}{2} \int_{0}^{L} \frac{T^{2}}{G J} d L=\frac{1}{2} \frac{T^{2} L}{G J}=\frac{1}{2} \frac{G J}{L}(\Delta \beta)^{2}
\end{aligned}
$$

Here, E, G, A, L, I, and J are respectively Young's modulus, shear modulus, crosssectional area, length, moment of inertia, and polar moment of inertia of the beam. On the other hand, $\Delta \mathrm{L}, \alpha$ and $\Delta \beta$ are respectively the length change, bending angle and torsional angle. If the energies are compared from Eqs. (2) - (4) in the molecular mechanics to Eqs. (5) - (7) in the structural mechanics, the following relations would be obtained:

$\frac{E A}{L}=K_{r}, \quad \frac{E I}{L}=K_{\theta}, \quad \frac{G J}{L}=K_{\tau}$

A, I and $\mathrm{J}$ are equal to $=\pi d^{2} / 4, I=\pi d^{2} / 64$, and $J=\pi d^{2} / 32$. Hence, the beam properties are obtained as follows $[42,43]$ :

$d=4 \sqrt{\frac{K_{\theta}}{K_{r}}}, \quad E=\frac{K_{r}^{2} L}{4 \pi K_{\theta}}, \quad G=\frac{K_{r}^{2} K_{\tau} L}{8 \pi K_{\theta}^{2}}$

The following relations are employed to evaluate the stiffnesses [44-46]:

$Y_{s}=\frac{8 \sqrt{3} K_{r}}{\frac{K_{r} r_{1}^{2}}{K_{\theta}}+18}$

$\vartheta=\frac{\frac{K_{r} r_{1}^{2}}{K_{\theta}}-6}{\frac{K_{r} r_{1}^{2}}{K_{\theta}}+18}$

$K_{\tau}=24 D$

where $Y_{s}, \vartheta$ and $D$ are surface (in-plane) Young's modulus, Poisson's ratio (the ratio of strain perpendicular to the loading direction to the strain along the loading direction) and flexural rigidity of the nanostructures. Moreover, $r_{1}$ is the length of bonds in the equilibrated structure. To obtain, $Y_{S}, \vartheta$ and $D$, DFT method is used. 


\section{Results and discussions}

\subsection{DFT Results}

For the range of $-0.05<\varepsilon<0.05$, variation of strain with respect to the strain energy for all nanotubes structures are shown Fig.3. Young's modulus is expressed by the following equation [47]:

$E=\frac{1}{V_{0}}\left(\frac{\partial^{2} E_{s}}{\partial \varepsilon^{2}}\right)_{\varepsilon_{=0}}$

where $\boldsymbol{V}_{\mathbf{0}}$ is the equilibrium volume of the unit-cell, $\mathrm{E}_{\mathrm{s}}$ is the strain energy and $\varepsilon$ is the

uniaxial strain. Therefore, computing the $\frac{\partial^{2} E_{S}}{\partial \varepsilon^{2}}$ from Fig. 3, Young's modulus are obtained for different structures as the values given in Table 2 .

Poisson's ratio can also be obtained by dividing the transverse strain to axial strain as presented below:

$\vartheta=-\frac{\varepsilon_{\text {trans. }}}{\varepsilon_{\text {axial }}}$

Computed values for Poisson's ratio of the considered nanotubes are also given in Table 2.

Fig. 4 depicts the stress-strain curves of the different considered nanotubes. As can be seen, at an equal tensile strain, CNT can tolerate significantly larger stress compared to other nanotubes. The same behavior is observed under compression. The smallest stress is associated with the SNNT. Furthermore, no important difference is observed in the tolerated stress by two other nanotubes.

\subsection{Flexural Rigidity}

If $E_{F}$ and $K$ are used to denote the strain energy per atom, $E_{F}$ and curvature of the structure, flexural rigidity is determined as [48]:

$D=\frac{\partial^{2} E_{F}}{\partial K^{2}}$

It should be noted that $K=1 / R$, and $R$ is the radius of curvature. Considering $E_{s h}$ and $E_{n}$ as the strain energy of unrolled (sheet) structure per atom and strain energy of rolled structure per atom, $E_{F}$ is obtained as [49]:

$E_{F}=E_{n}-E_{s h}$ 
The strain energy per atom of CNT, SiNT, GeNT and SnNT are plotted in Fig.5 against

K. Computing $\frac{\partial^{2} E_{F}}{\partial K^{2}}$ from this curves and substituting it into Eq. (15), flexural rigidity can be obtained. The resulted values are given in Table 2.

Bond stiffnesses are computed for nanotubes by replacing the values of $Y_{S}, \vartheta$ and $D$ from Table 2 into Eqs. (10)-(12). In this way, stiffnesses are obtained as the values given in Table 3.

Now beam properties are computed for nanotubes by substituting the bond stiffness which are given in Table 3 into Eq. (9). Hence, the beam properties for FE model are obtained as the values given in Table 4 .

\subsection{FE results}

As same as DFT modeling, the $(10,10)$ armchair nanotubes of CNT, SiNT, GeNT and SnNT are selected to model in ABAQUS 6.14 software. The length and diameter for each of them are shown in Table 5. In this approach, to obtain Young's modulus of pristine NTs of group IV, all of degrees of freedom are restrained at one side and the strain is applied to the opposite side. Boundary condition and applied strain on the nanotubes are shown in Fig.5. To compute the elastic modulus, following equation is used:

$E_{N T}=\frac{\sigma}{\varepsilon}=\frac{\frac{F}{A_{0}}}{\frac{\Delta L}{L_{0}}}$

where $\mathrm{F}$ is the force applied over the cross-sectional area $\varepsilon$ is applied strain , $\Delta L$ length change, $L_{0}$ initial length and $A_{0}$ is the area of the nanotube cross section before the loading and is stated as:

$A_{0}=\pi D t$

where $D$ and $\mathrm{t}$ are mean diameter and thickness of the NT, respectively. The values of Young's modulus of nanotubes that obtaining by FE method are given in Table 5.

As it can be seen, its accuracy is acceptable compared with DFT method. Therefore FE method can be a reliable and accurate substitute for the density functional method. Due to the good accuracy of the Young's modulus calculated by the FE method. It can be concluded that the FE method can be used to obtain the mechanical properties of the simulated nanotubes. The Young's modulus of group-IV armchair nanotubes in range of $(3,3)$ up to $(15,15)$ are 
depicted in Fig. 7 to compare. It can be concluded that the FE method can be used to obtain the mechanical characteristics of the simulated nanotubes.

The results show that by increasing the radius of nanotubes at the same length leads to increasing Young's modulus of the nanotube. Besides, Young's modulus decreases by increasing the bond length. There is a great difference between the Young's modulus of CNT and the other nanotube including SiNT, GeNT and SnNT. As can be seen in Fig. 7, the Young's modulus of CNTs at least 10 times larger than other nanotubes at the same chirality. For example the Young's modulus of $(5,5)$ armchair CNT, SiNT, GeNT and SnNT are 1021, 106.4, 76.16 and 70.87GPa respectively.

\section{Conclusion}

In this study, the mechanical properties of $(10,10)$ armchair CNT, SiNT, GeNT and SnNT were calculated by DFT method. Young's modulus, Poisson's ratio, and flexural rigidity of the pristine nanotube of group IV of periodic table were calculated from the DFT calculations. Then, they were employed to compute the properties of beam elements for FE simulation models. In the next step, a FE model was proposed base on DFT model. Results of FE and DFT methods were compared and it is found that the developed FE model is able to predict the elastic characteristics of the nanotubes accurately.

\section{Declarations}

Funding: This research received no specific grant from any funding agency in the public, commercial, or not-for-profit sectors.

Conflicts of interest/Competing interests: The authors declare that they have no conflict of interest/Competing interests.

Ethics approval: $\quad$ N/A

Consent to participate: N/A

Consent for publication: N/A

Availability of data and material: The raw/processed data required to reproduce these findings cannot be shared at this time due to technical or time limitations.

Code availability: The code required to reproduce these findings cannot be shared at this time due to technical or time limitations. 


\section{References}

[1] S. Iijima, Nature (London) 354 (1991) 56].

[2] Bethune, D. S.; Kiang, C. H.; de Vries, M. S.; Gorman, C.; Savoy, R.; Vazquez, J.; Beyers, R. Cobalt-Catalyzed Growth of Carbon Nanotubes with Single Atomic Layer Walls. Nature 1993, 363, 605-607.

[3] Iijima, S.; Ichihashi, T. Single Shell Carbon Nanotubes of 1-nm Diameter. Nature 1993, 363, 603-605.

[4] V.N. Popov, Mater. Sci. Eng. R 43 (2004) 61

[5] M.M.J. Treacy, T.W. Ebbesen, J.M. Gibson, Nature (London) 381 (1996) 678.

[6] A. Krishnan, E. Dujardin, T.W. Ebbesen, P.N. Yianilos, M.M.J. Treacy, Phys. Rev. B 58 (1998) 14013.

[7] J.-P. Salvetat, G.A.D. Briggs, J.-M. Bonard, R.R. Bacsa, A.J. Kulik, T. Stockli, N.A. Burnham, L. Forro, Phys. Rev. Lett. 82 (1999) 944.

[8] E. Hernandez, C. Goze, P. Bernier, A. Rubio, Phys. Rev. Lett. 80 (1998) 4502.

[9] G. Van Lier, C. Van Alsenoy, V. Van Doren, P. Geerlings, Chem. Phys. Lett. 326 (2000) 181.

[10] E. Hernandez, C. Goze, P. Berneir, A. Rubio, Appl. Phys. A Mater. Sci. Process. 68 (1999) 287.

[11] J.M. Molina, S.S. Savinsky, N.V. Khokhriakov, J. Chem. Phys. 104 (1996) 4652.

[12] K.I. Tsepes, P. Papanikos, Finite Element Modeling of Single walled Carbon Nanotubes.Compos. B Eng. 36 (2005) 468-477.

[13] A.L. Kalamkarvo, A.V. Georgiades, S.K. Rokkam, V.P. Veedu, M.N. Ghasemi-Nejhad, Analytical and Numerical Techniques to Predict Carbon Nanotubes Properties. Int. J. Solids Struct. 43 (2006) 6832-6854.

[14] A. Ebrahimi, H. Ehteshami, M. Mohammadi, Density functional calculations of response of single-walled armchair carbon nanotubes to axial tension. Computational Materials Science 41 (2008) 486-492. 
[15] Jin-Liang Zang, Quanzi Yuan, Feng-Chao Wang, Ya-Pu Zhao, A comparative study of Young's modulus of single-walled carbon nanotube by CPMD, MD and first principle simulations, Computational Materials Science 46 (2009) 621-625.

[16] Roham Rafiee, Meghdad Heidarhaei, Investigation of chirality and diameter effects on the Young's modulus

of carbon nanotubes using non-linear potentials, Composite Structures 94 (2012) 2460-2464.

[17] M. M. Zaeri, S. Ziaei-Rad, A. R. Shahidi, On the Elastic Constants of Single Walled Carbon Nanotubes, Procedia Materials Science 11 (2015) 666 - 671.

[18] Nguyen Tuan Hung, Do Van Truong, Vuong Van Thanh, Riichiro Saito, Intrinsic strength and failure behaviors of ultra-small single-walled carbon nanotubes, Computational Materials Science 114 (2016) 167-171.

[19] Novoselov, Kostya S., Andre K. Geim, Sergei V. Morozov, D. Jiang, Y_ Zhang, Sergey V. Dubonos, Irina V. Grigorieva, and Alexandr A. Firsov. "Electric field effect in atomically thin carbon films." science 306, no. 5696 (2004): 666-669.

[20] S. Cahangirov, M. Topsakal, E. Akturk, H. Sahin, and S. Ciraci, “ Phys. Rev. Lett. 102, 236804 (2009).

[21] G. G. Guzman-Verri and L. C. Lew Yan Voon,' Phys. Rev. B 76, 075131 (2007).

[22] F. Bechstedt, L. Matthes, P. Gori, and O. Pulci, Appl. Phys. Lett. 100, 261906 (2012).

[23] L. Matthes, P. Gori, O. Pulci, and F. Bechstedt, Phys. Rev. B 87, 035438 (2013).

[24] L. Matthes, O. Pulci, and F. Bechstedt,J. Phys.: Condens. Matter 25, 395305 (2013).

[25] Y. Xu, B. Yan, H. J. Zhang, J. Wang, G. Xu, P. Tang, W. Duan, and S. C. Zhang, Phys. Rev. Lett. 111, 136804 (2013).

[26] B. V. D. Broek, M. Houssa, E. Scalise, G. Pourtois, V. V. Afanasev, and A. Stesmans, 2D Mater. 1, 021004 (2014)

[27] S. Balendhran, S. Walia, H. Nili, S. Sriram, and M. Bhaskaran, Small 11, 640 (2015)

[28] Bohayra Mortazavi, Obaidur Rahaman, Meysam Makaremi, Arezoo Dianat, Firstprinciples investigation of mechanical properties of silicene, germanene and stanine. Physica E 87 (2017) 228-232.

[29] Ki Ryang Byun, Jeong Won Kang and Ho Jung Hwang, Atomistic Simulation of Hypothetical Silicon Nanotubes under Tension, Journal of the Korean Physical Society, Vol. 42, No. 5, (2003), 635-646.

[30] Veena Verma, K. Dharamvir and V. K. Jindal, Structure and Elastic Modulii of Silicon Nanotubes, Journal of Nano Research Vol 2 (2008) 85-90. 
[31] A.R. Setoodeh, H. Attariani, M. Jahanshahi, Mechanical Properties of SiliconGermanium Nanotubes under Tensile and Compressive Loadings, Journal of Nano Research Vol 15 (2011) 105-114.

[32] Amirali Abbasi and Jaber Jahanbin Sardroodi, Structural and electronic properties of group-IV tin nanotubes and their effects on the adsorption of SO2 molecules: Insights from DFT computations, JOURNAL OF APPLIED PHYSICS 124 (2018) 165302.

[33] J.P. Perdew, K. Burke, M. Ernzerhof, Phys. Rev. Lett. 77 (1996) 3865.

[34] J.P. Perdew, K. Burke, Y. Wang, Phys. Rev. B 54 (1996) 16533.

[35] Lars Matthes, Olivia Pulci and Friedhelm Bechstedt, Massive Dirac quasiparticles in the optical absorbance of graphene, silicene, germanene, and tinene, J. Phys.: Condens. Matter 25 (2013) 395305.

[36 Odegard GM, Gates TS, Nicholson LM, Wise KE. Equivalent continuum modelling of nano-structured materials. Compos Sci Technol 2002; 62(14):1869-1880.

[37] Li C, Chou TW. Elastic moduli of multi-walled carbon nanotubes and the effect of van der Waals forces. Compos Sci Technol 2003; 63 (11): 1517-1524.

[38] Rappe AK, Casemit CJ, Colwell KS, Goddard WA, Skiff WM. UFF, a full periodic table force-field for molecular mechanics and molecular dynamics simulations. J Am Chem Soc 1992; 114 (25): 10024-10035.

[39] C. Li, T.W. Chou, A structural mechanics approach for the analysis of carbon nanotubes, Int. J. Solids Struct. 40 (2003) 2487-2499.

[40] B.R. Gelin, Molecular modeling of polymer structures and properties, Hanser Publ.; Hanser/Gardner Publ. (1994).

[41] T. Chang, H. Gao, Size-dependent elastic properties of a single-walled carbon nanotube via a molecular mechanics' model, J. Mech. Phys. Solids 51 (2003) 1059-1074

[42] R. Ansari, S. Rouhi, Atomistic finite element model for axial buckling of single walled carbon nanotubes, Physica E: Low. -Dimens. Syst. Nanostruct. 43 (2010) 58-69.

[43] S. Rouhi, R. Ansari, Atomistic finite element model for axial buckling and vibration analysis of single-layered graphene sheets, Physica E: Low. -Dimens. Syst. Nanostruct. 44 (2012) 764-772.

[44] R. Ansari, S. Rouhi, M. Aryayi, M. Mirnezhad, On the buckling behavior of single walled silicon carbide nanotubes, ScientiaIranica 19 (2012) 1984-1990.

[45] R. Ansari, S. Rouhi, M. Mirnezhad, M. Aryayi, Stability characteristics of single layered silicon carbide nanosheets under uniaxial compression, Physica E: Low. - Dimens. Syst. Nanostruct. 53 (2013) 22-28. 
[46] R. Ansari, S. Rouhi, M. Mirnezhad, M. Aryayi, Stability characteristics of single walled boron nitride nanotubes, Arch. Civ. Mech. Eng. 15 (2015) 162-170.

[47] Douglas Vodnik, The Young's Modulus of Single-Walled Carbon Nanotubes, Department of Physics, Carthage College, Kenosha, WI

[48] R. Ansari, S. Rouhi, M. Mirnezhad, M. Aryayi, Stability characteristics of single walled boron nitride nanotubes, Arch. Civ. Mech. Eng. 15 (2015) 162-170.

[49] R. Ansari, S. Rouhi, M. Mirnezhad, M. Aryayi, Stability characteristics of single walled boron nitride nanotubes, Arch. Civ. Mech. Eng. 15 (2015) 162-170.

\section{Table Captions}

Table 1. Structural and electronic parameters for group IV elements [35]

Table 2. Young's Modulus, Poisson's Ratio and flexural rigidity of group IV nanotube

Table 3. Bond stiffnesses for group IV nanotubes

Table 4. Properties of beam elements for group IV nanotubes

Table 5. Young's modulus of group IV $(10,10)$ armchair nanotubes obtained by FE method and comparing with DFT

\section{Figure Captions}

Fig. 1. Schematics of the simulated unit cells for different group IV nanotubes. $(10,10)$ armchair nanotubes with 40 atoms are considered.

Fig. 2. Representation of the bond length (d) and buckling height $(\delta)$

Fig. 3. Strain energy versus strain for $(10,10)$ armchair nanotubes

Fig. 4. Stress-strain curves of the considered nanotubes

Fig. 5. Flexural rigidity CNT, SiNT, GeNT and SnNT

Fig. 6. Loading conditions to obtain Young's modulus of nanotubes using the proposed FE model.

Fig. 7. Young's modulus of CNT, SiNT, GeNT and SnNT against diameter 


\section{Tables}

Table 1. Structural and electronic parameters for group IV elements [35]

\begin{tabular}{ccccc}
\hline & $\mathrm{C}$ & $\mathrm{Si}$ & $\mathrm{Ge}$ & $\mathrm{Sn}$ \\
$a\left(\mathrm{~A}^{\circ}\right)$ & 2.468 & 3.868 & 4.060 & 4.673 \\
$d\left(\mathrm{~A}^{\circ}\right)$ & 1.425 & 2.233 & 2.344 & 2.698 \\
$\delta$ & 0.00 & 0.45 & 0.69 & 0.85
\end{tabular}

a: Lattice constant

d: In-plane nearest-neighbor distance

$\delta$ : Buckling height

Table 2. Young's Modulus, Poisson's Ratio and flexural rigidity of group IV nanotube

\begin{tabular}{ccccc}
\hline & CNT & SiNT & GeNT & SnNT \\
Young's Modulus(GPa) & 1029 & 159.82 & 83.23 & 83.15 \\
Poisson's Ratio & 0.27 & 0.34 & 0.35 & 0.44 \\
Flexural Rigidity (D) & 1.97265 & 3.62909 & 1.50843 & 2.28517 \\
\hline
\end{tabular}

Table 3. Bond stiffnesses for group IV nanotubes

\begin{tabular}{cccc}
\hline Nanotube & $k_{r}\left(\frac{n N}{n m}\right)$ & $k_{\theta}(n N . n m)$ & $k_{\tau}(n N . n m)$ \\
\hline CNT & $8.335 \mathrm{E}-7$ & $1.121 \mathrm{E}-9$ & $2.787 \mathrm{E}-10$ \\
SiNT & $1.627 \mathrm{E}-7$ & $4.419 \mathrm{E}-9$ & $5.128 \mathrm{E}-10$ \\
GeNT & $1.220 \mathrm{E}-7$ & $3.542 \mathrm{E}-9$ & $2.132 \mathrm{E}-10$ \\
SnNT & $8.487 \mathrm{E}-7$ & $2.485 \mathrm{E}-9$ & $3.229 \mathrm{E}-10$ \\
\hline
\end{tabular}


Table 4. Properties of beam elements for group IV nanotubes

\begin{tabular}{cccc}
\hline Nanotube & $d(\mathrm{~nm})$ & $E(\mathrm{TPa})$ & $G(\mathrm{TPa})$ \\
\hline CNT & 0.146 & 7.008 & 0.871 \\
SiNT & 0.208 & 10.650 & 0.618 \\
GeNT & 0.215 & 7.836 & 0.236 \\
SnNT & 0.216 & 6.222 & 0.404
\end{tabular}

Table 5. Young's modulus of group IV $(10,10)$ armchair nanotubes obtained by FE method and comparing with DFT

\begin{tabular}{|c|c|c|c|c|c|}
\hline \multirow{3}{*}{ Nanotube Type } & \multirow{3}{*}{$\begin{array}{c}\text { Average } \\
\text { Diameter }\left(\mathrm{A}^{0}\right)\end{array}$} & \multirow{3}{*}{ Length $\left(A^{0}\right)$} & \multicolumn{2}{|c|}{$\mathrm{E}_{\mathrm{NT}}(\mathrm{GPa})$} & \multirow{3}{*}{ Error(\%) } \\
\hline & & & $\mathrm{FE}$ & DFT & \\
\hline & & & Method & Method & \\
\hline CNT & 6.78 & 23.39 & 1090 & 1029 & 5.5 \\
\hline SiNT & 10.66 & 36.74 & 154.67 & 159.82 & 0.6 \\
\hline GeNT & 11.19 & 38.57 & 85.2 & 83.23 & 2.3 \\
\hline SnNT & 12.88 & 44.39 & 82.6 & 83.15 & 0.6 \\
\hline
\end{tabular}




\section{Figures}
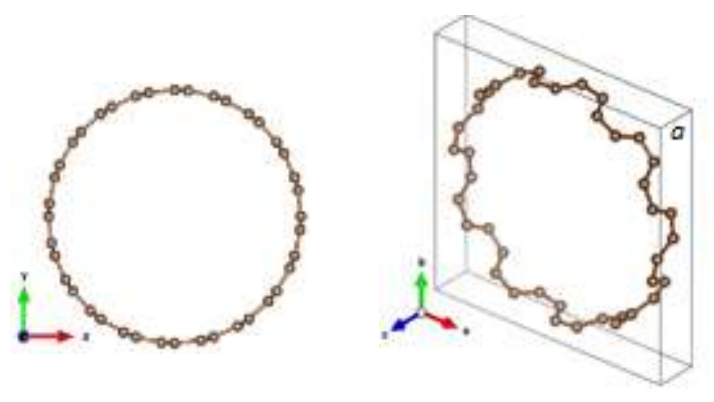

(a) $\mathrm{CNT}$

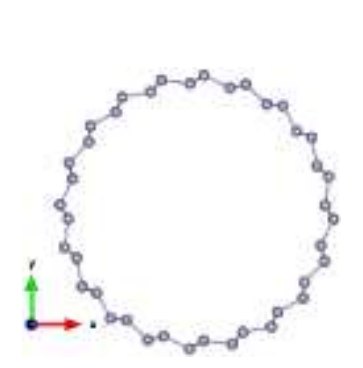

(c) GeNT

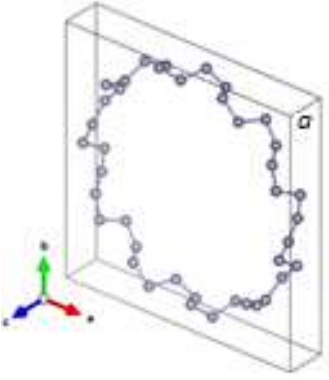

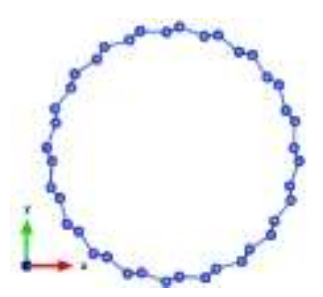

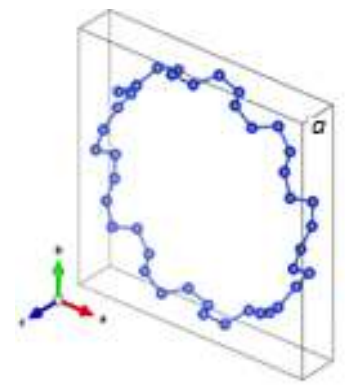

(b) SiNT
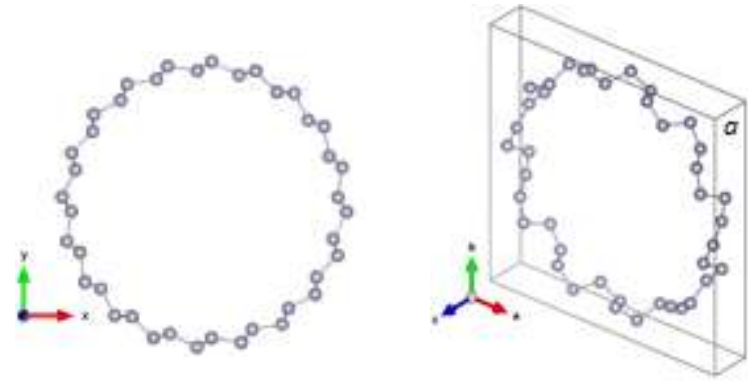

(d) SnNT

Fig. 1. Schematics of the simulated unit cells for different group IV nanotubes. $(10,10)$ armchair nanotubes with 40 atoms are considered.

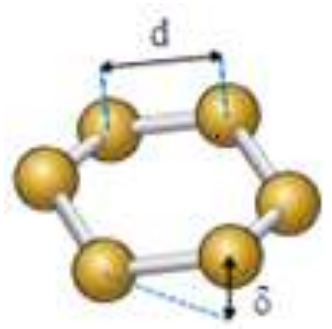

Fig. 2. Representation of the bond length (d) and buckling height $(\delta)$ 


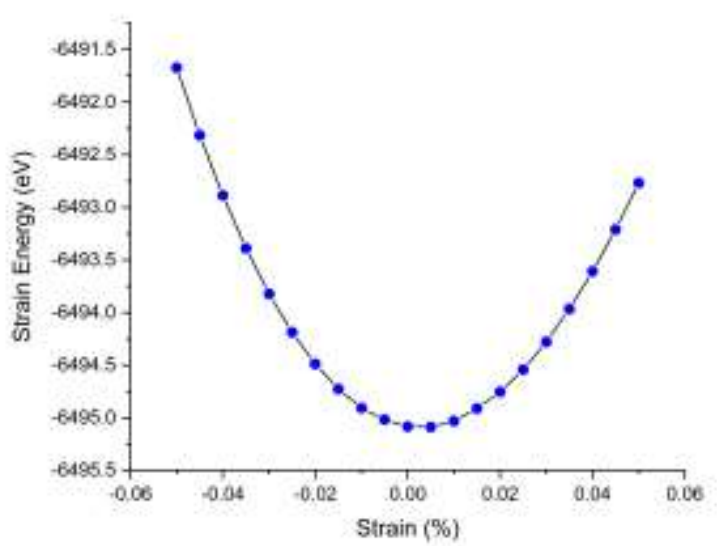

(a) $\mathrm{CNT}$

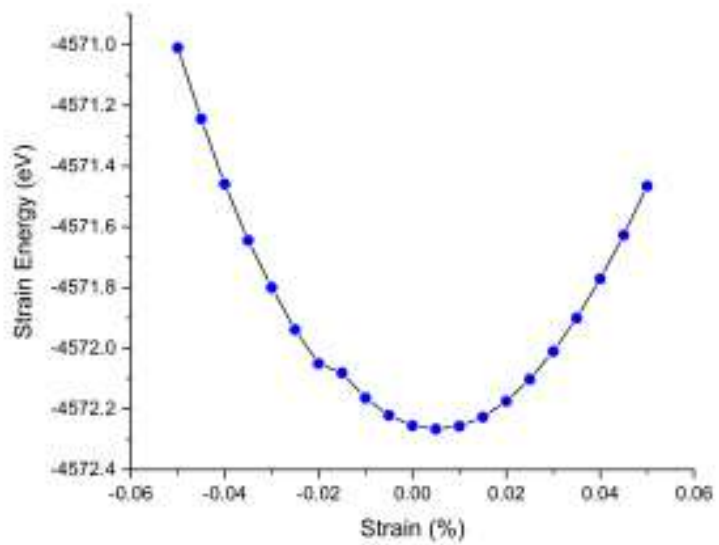

(c) GeNT

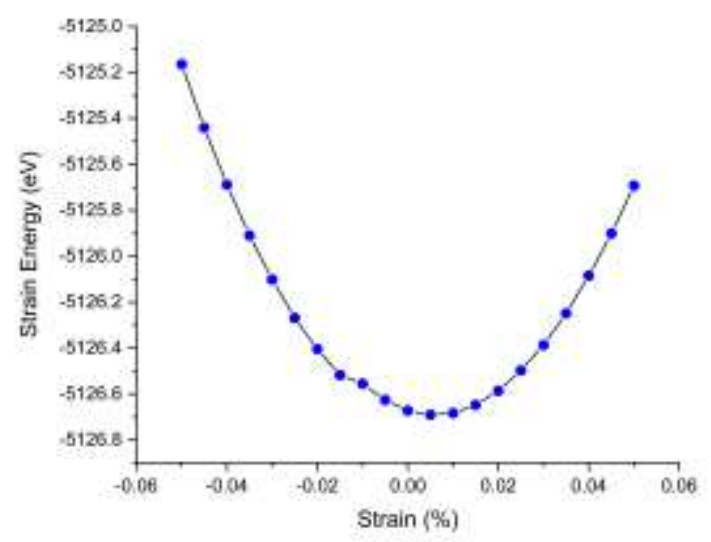

(b) SiNT

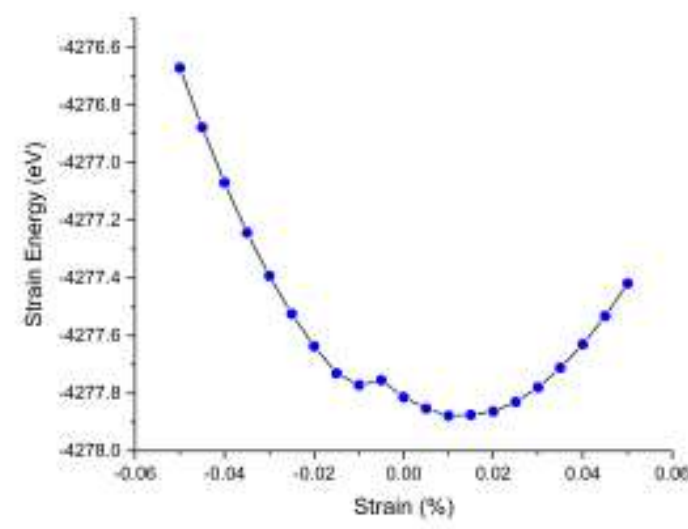

(d) SnNT

Fig. 3. Strain energy versus strain for $(10,10)$ armchair nanotubes 


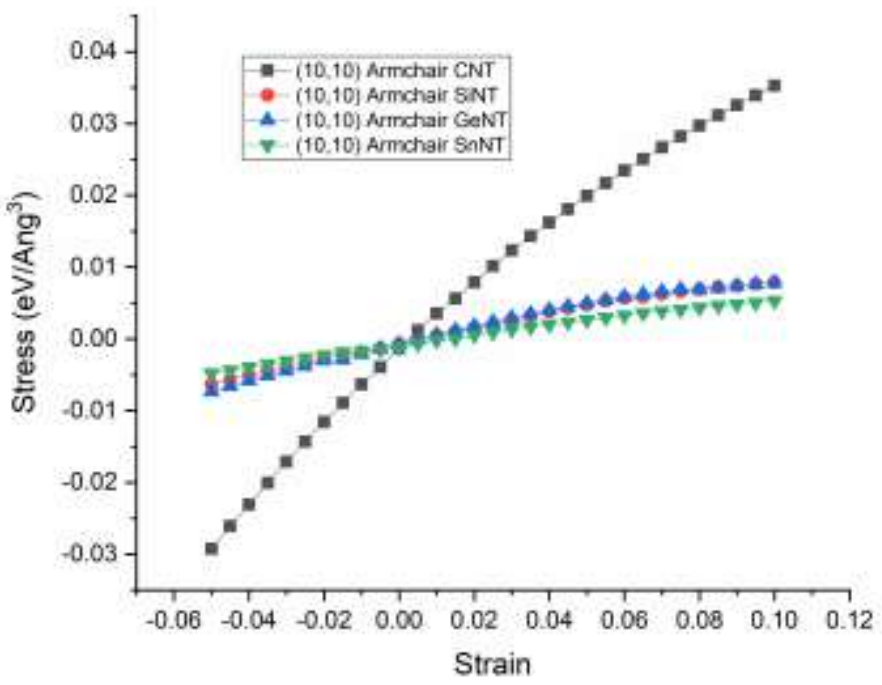

Fig. 4. Stress-strain curves of the considered nanotubes 


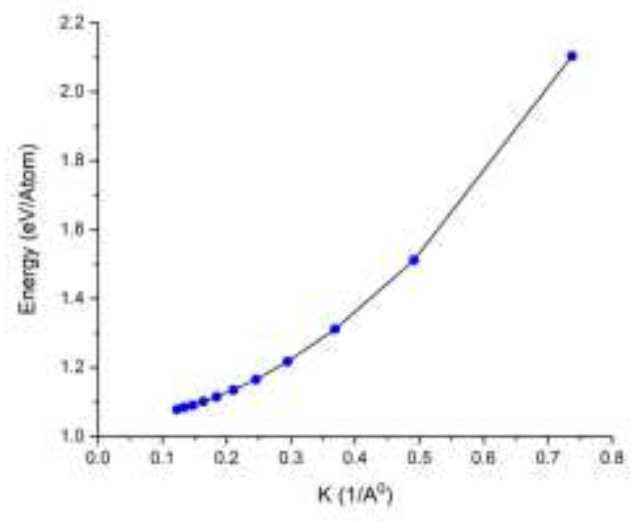

(a) $\mathrm{CNT}$

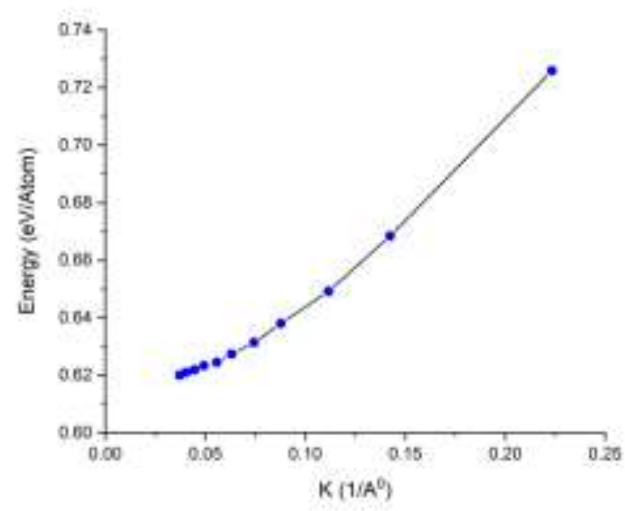

(c) GeNT

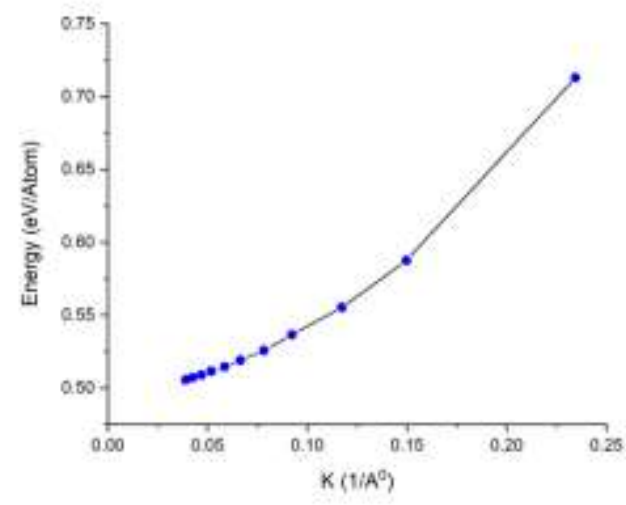

(b) SiNT

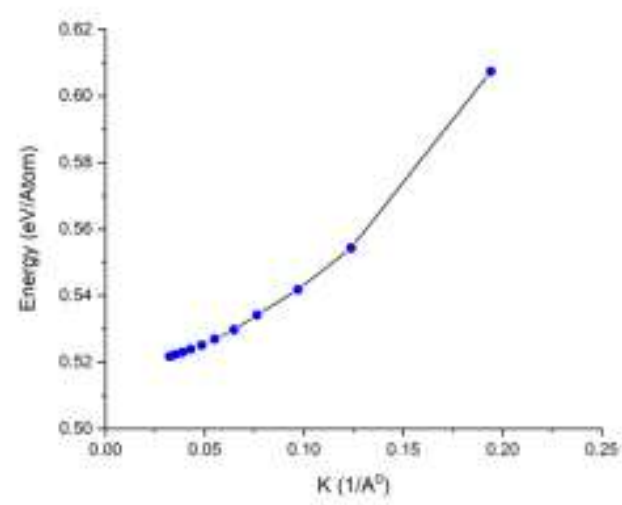

(d) SnNT

Fig. 5. Flexural rigidity CNT, SiNT, GeNT and SnNT 

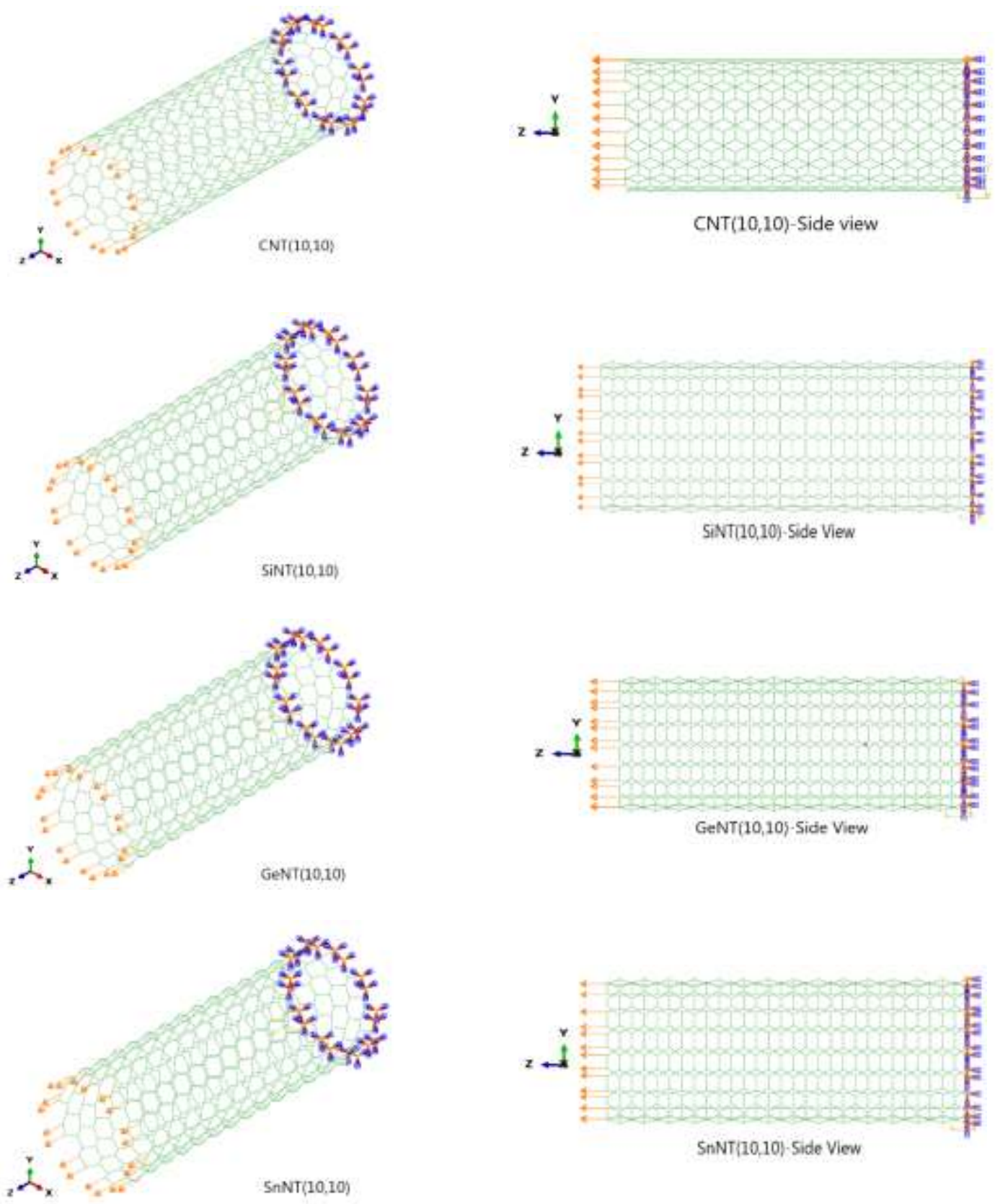

Fig. 6. Loading conditions to obtain Young's modulus of nanotubes using the proposed FE model. 


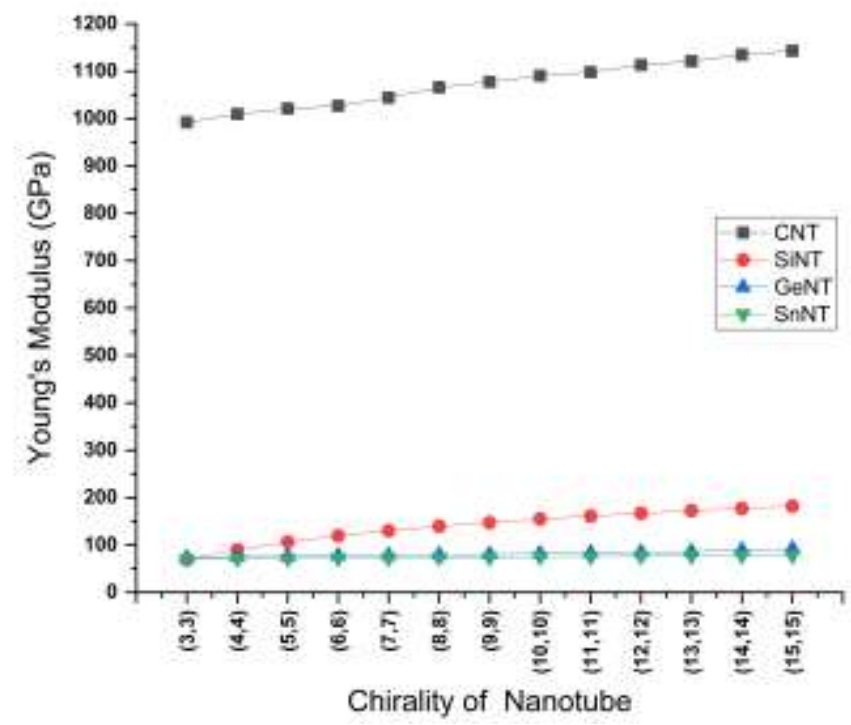

Fig. 7. Young's modulus of CNT, SiNT, GeNT and SnNT against diameter 
Figures
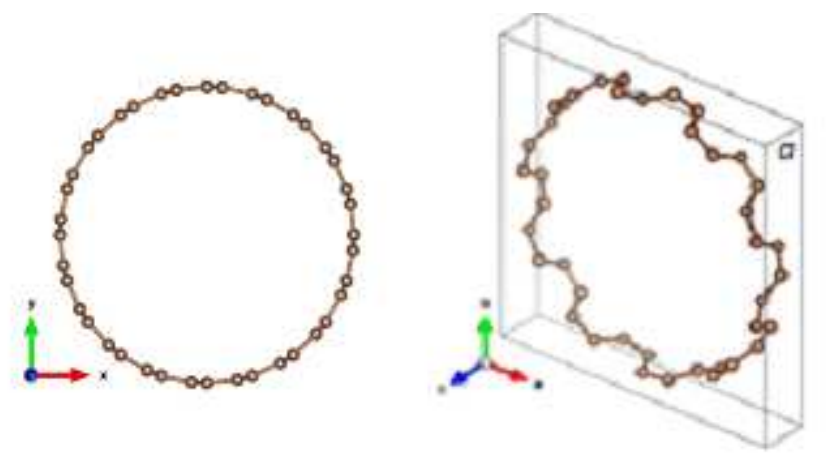

(a) $\mathrm{CNT}$
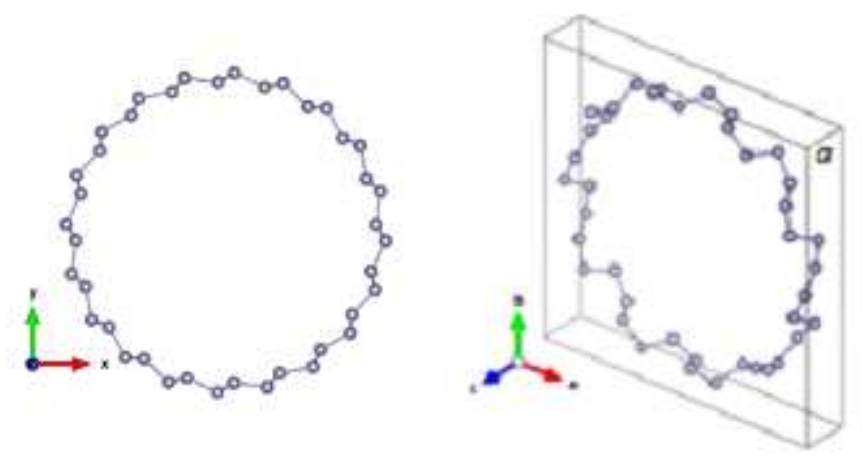

(c) GeNT
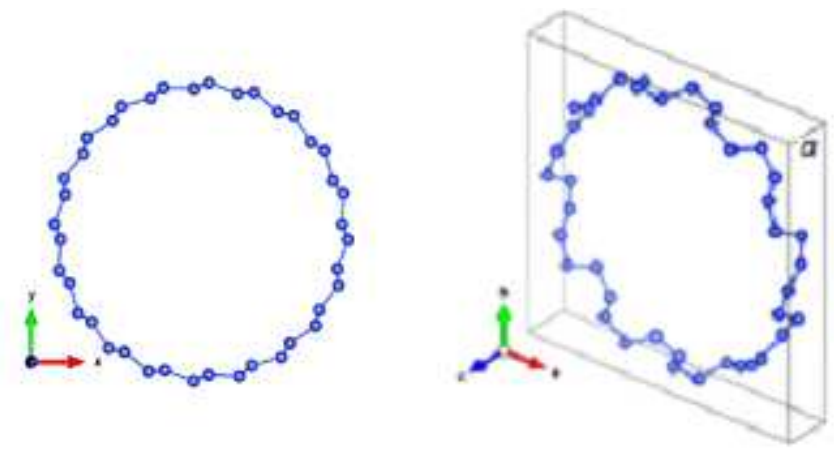

(b) SiNT
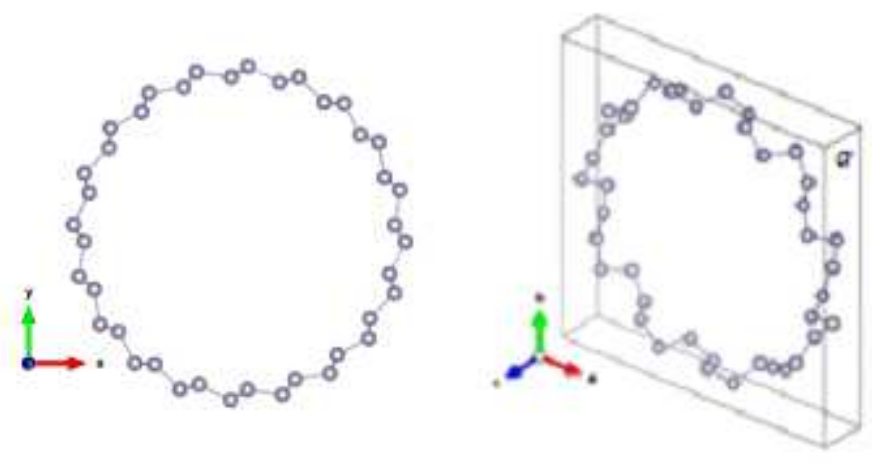

(d) $\mathrm{SnNT}$

\section{Figure 1}

Schematics of the simulated unit cells for different group IV nanotubes. $(10,10)$ armchair nanotubes with 40 atoms are considered. 


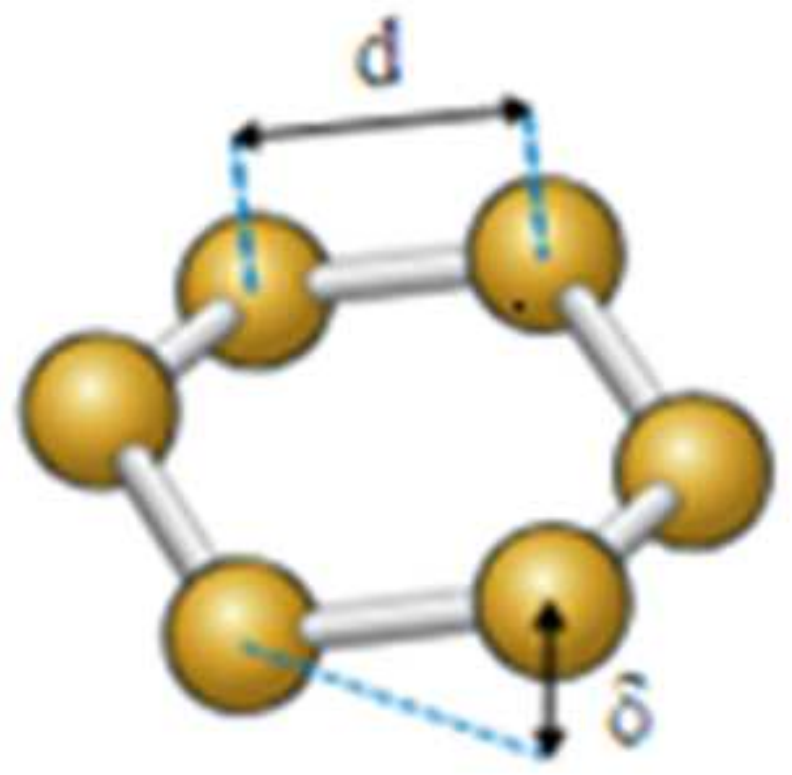

Figure 2

Representation of the bond length (d) and buckling height ( $\delta$ ) 


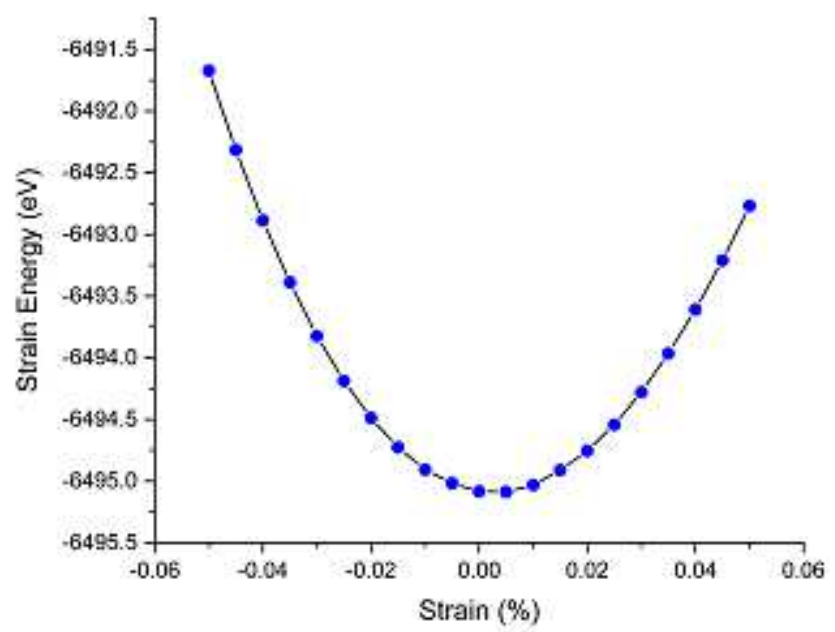

(a) $\mathrm{CNT}$

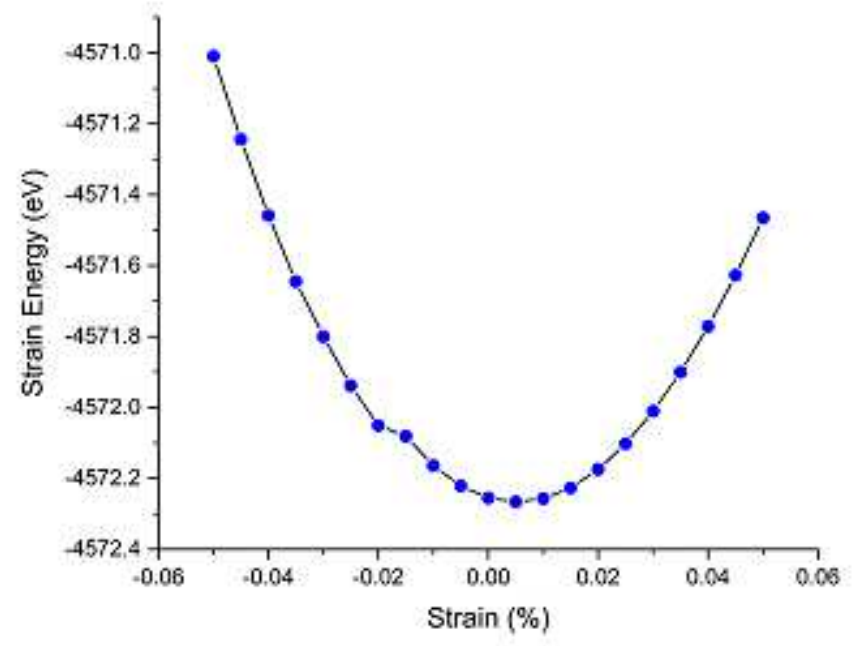

(c) GeNT

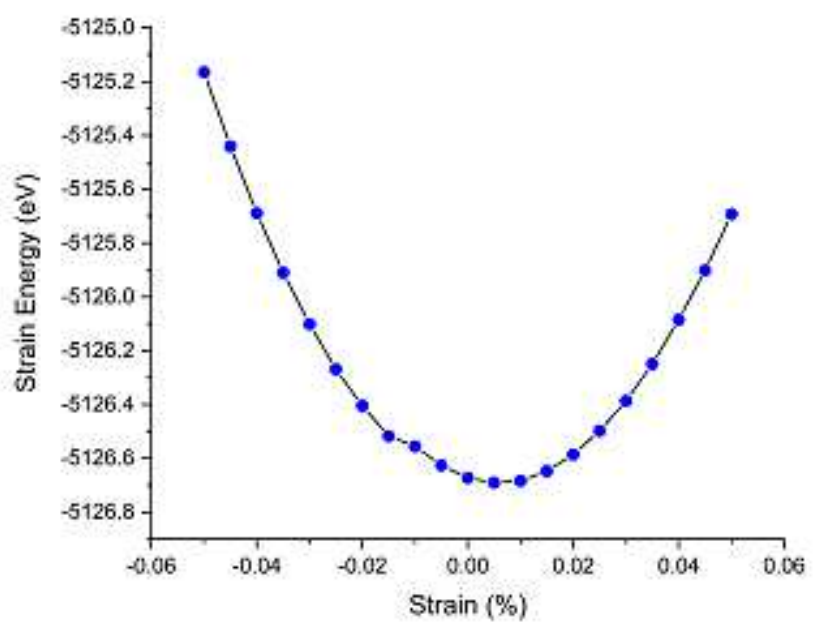

(b) SiNT

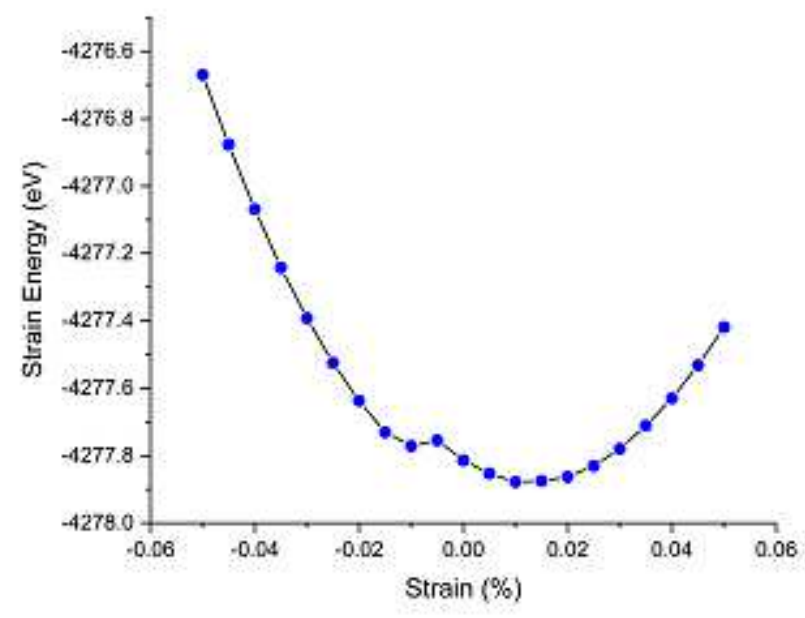

(d) $\mathrm{SnNT}$

\section{Figure 3}

Strain energy versus strain for $(10,10)$ armchair nanotubes 


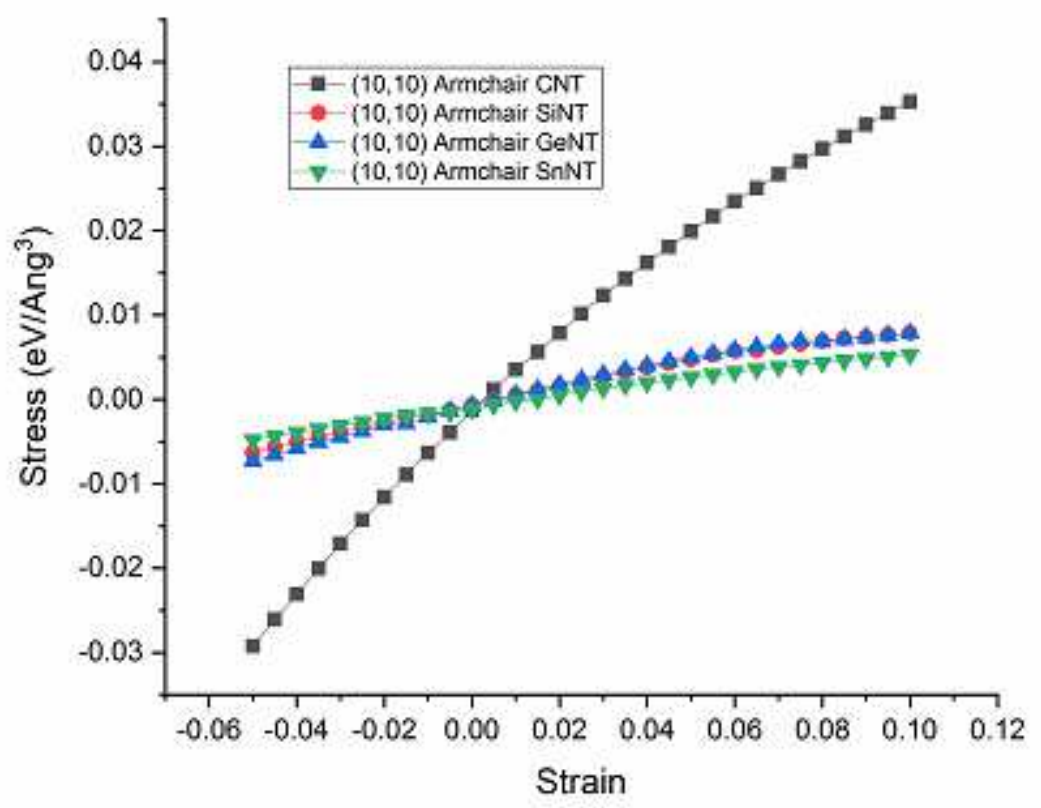

Figure 4

Stress-strain curves of the considered nanotubes 


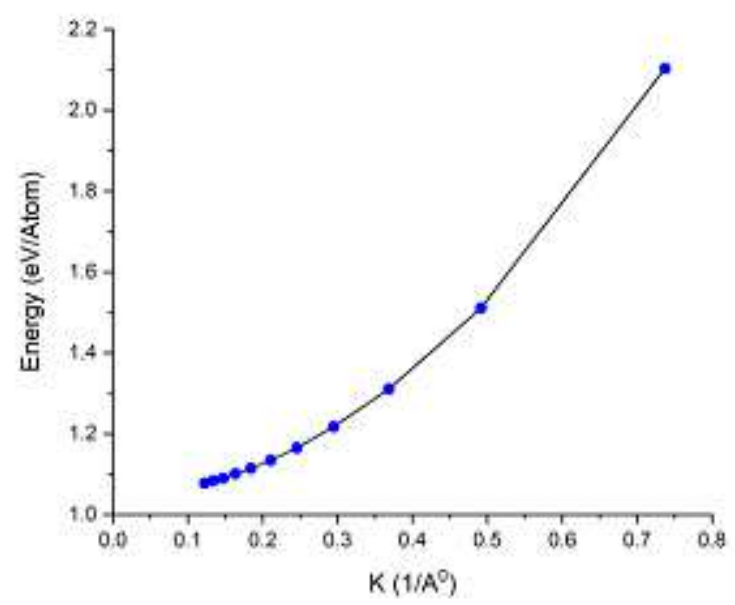

(a) CNT

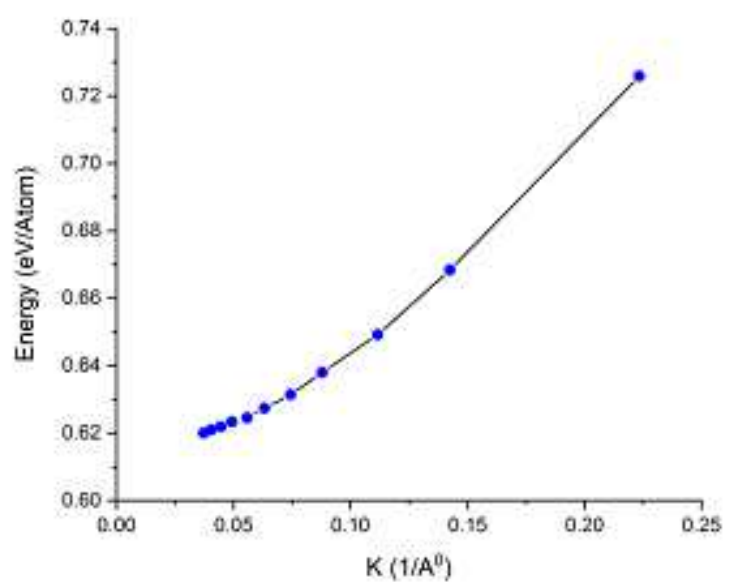

(c) GeNT

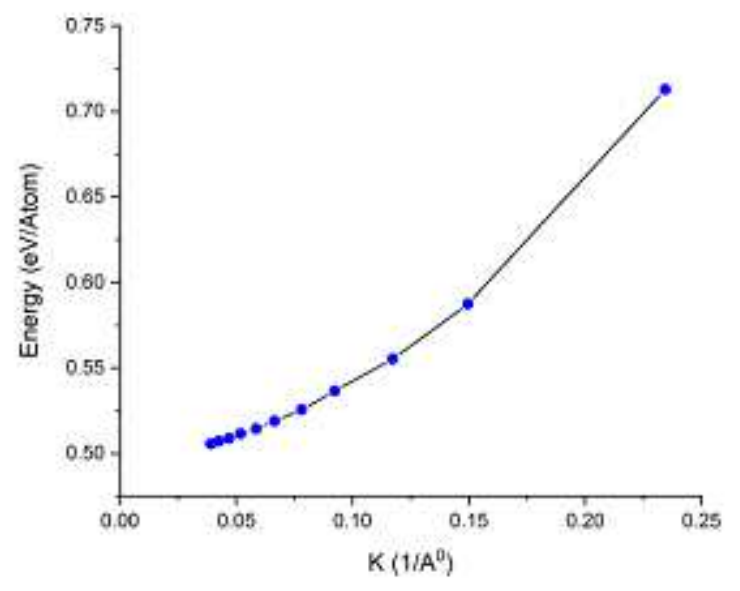

(b) SiNT

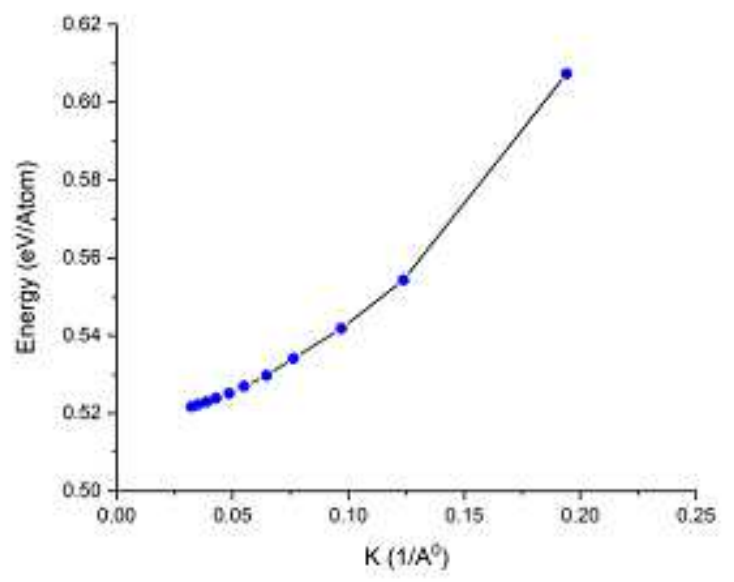

(d) $\mathrm{SnNT}$

Figure 5

Flexural rigidity CNT, SiNT, GeNT and SnNT 

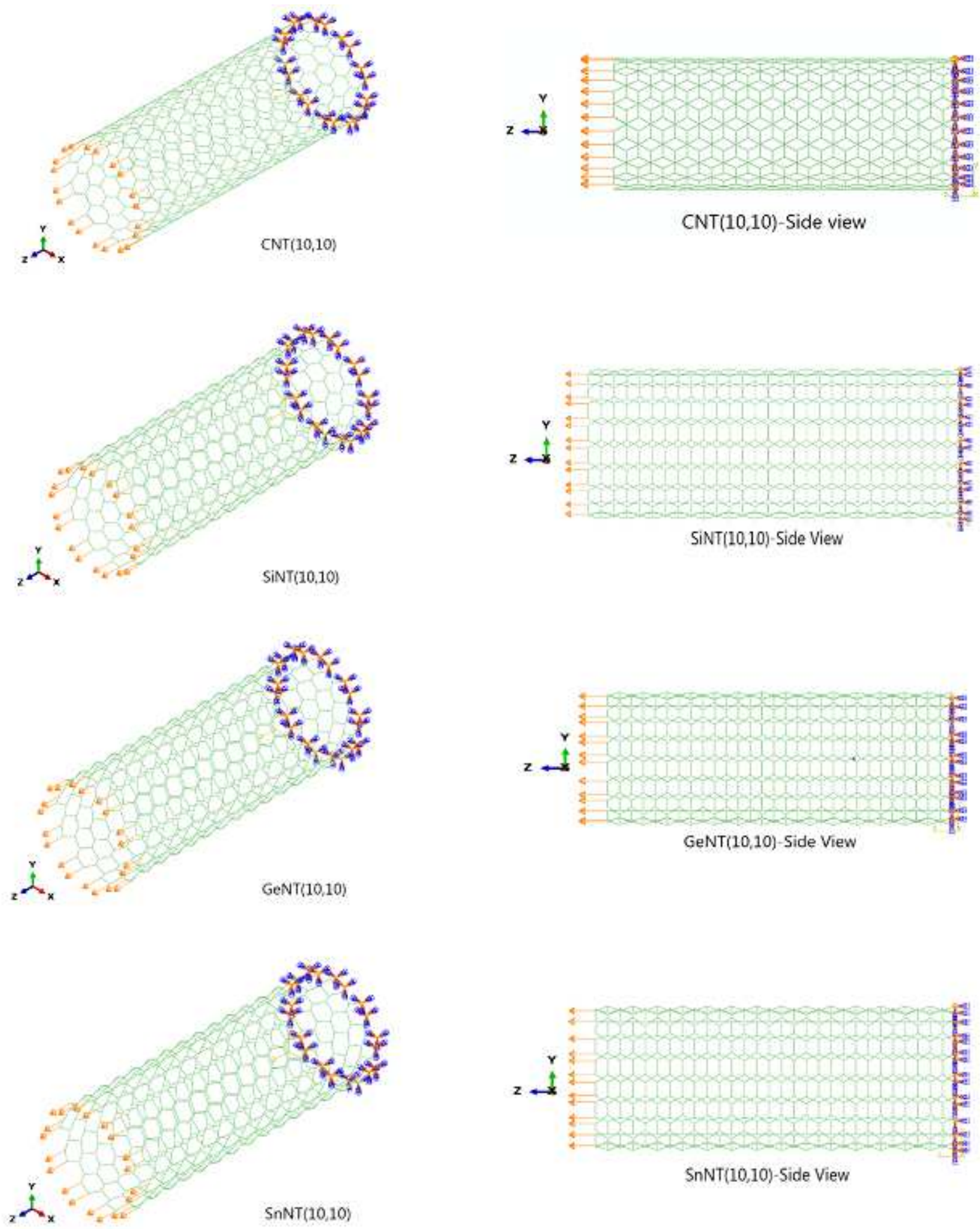

Figure 6

Loading conditions to obtain Young's modulus of nanotubes using the proposed FE model. 


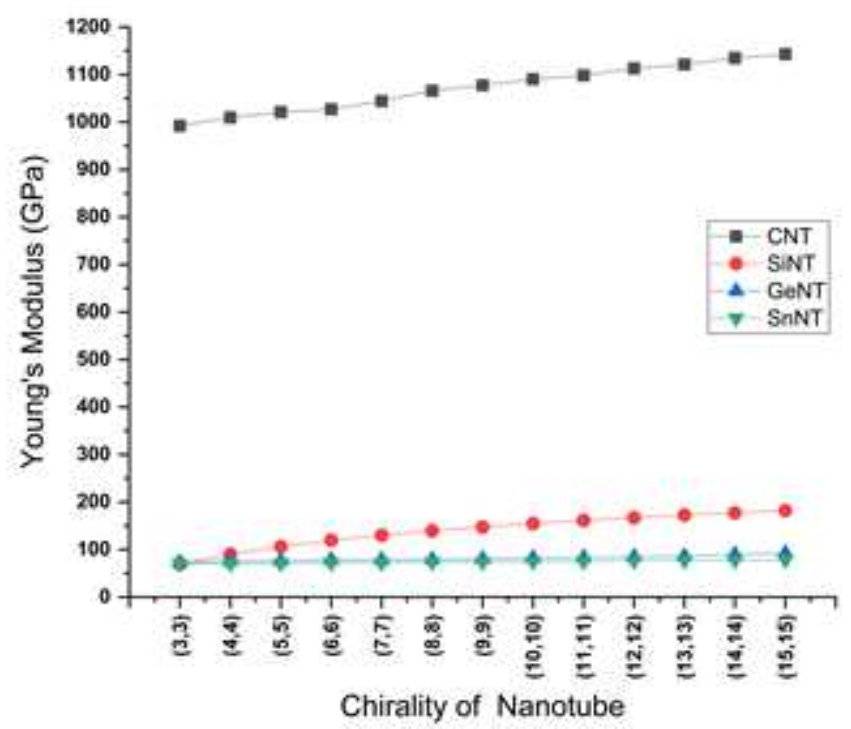

Figure 7

Young's modulus of CNT, SiNT, GeNT and SnNT against diameter 